\title{
Ethical considerations in managing the blast flag in automated hematology analyzers
}

\section{Ozgur Aydin}

Department of Biochemistry Laboratory, Kepez Public Hospital, Antalya, Turkey

\section{Dear Editor,}

A 9-year-old Syrian refugee girl presented at the hospital with a complaint of abdominal discomfort. The physical examination revealed multiple enlarged lymph nodes and hepatosplenomegaly. A complete blood count (CBC) showed a $70 \%$ lymphoid predominance, while the white blood cell, red blood cell, and platelet values were within the normal ranges. Her pediatrician requested a blood smear with a pre-diagnosis of infectious mononucleosis to rule out malignancy. A peripheral blood smear was prepared from a drop of blood from the ethylenediamine tetraacetic acid sample and stained as described.

On the blood smear, lymphoid predominance was quite obvious. Large lymphocytes with large, irregular nuclei were easily visible (Fig. 1). The lymphocytes were clearly atypical, but the features fell short of the definition of a blast. I decided to check the instrument results, where I saw "blasts" and "atypical lymphoid cells" flags (Fig. 1). The analyzer had detected the atypical cells and created an alarm flag. Unfortunately, these instrument flags are not sent to the laboratory information system (LIS), so while validating test results this information will not typically be available. My pediatrician colleague and I accepted the flag as an extra warning to refer the patient to a hematology center. On follow-up, the diagnosis was infectious mononucleosis based on a physical examination and positive Epstein-Barr virus immunoglobulin (Ig)M and IgG results.

Most automated hematology analyzers generate blast flags. The instrument manuals recommend a blood smear examination to confirm these warnings. On a Tuesday in mid-October

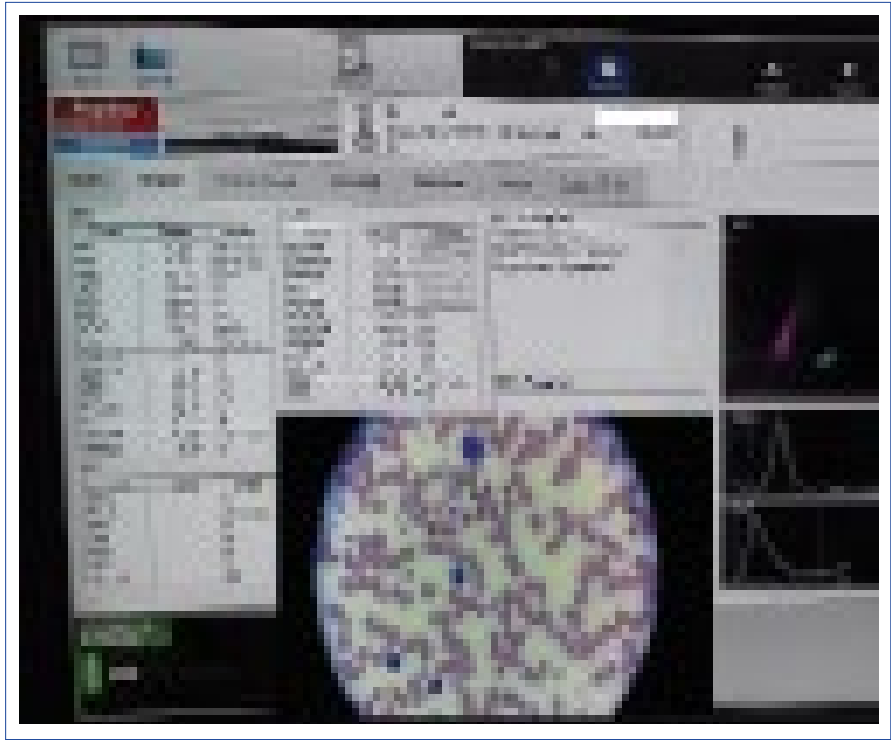

Figure 1. Sysmex XN 1000 instrument (Sysmex Corp., Kobe, Japan) results page is seen. The results of the patient were presented with "Blasts" and "Atypical Lymphocytes" flags. In the peripheral blood smear (inlet) some atypical lymphocytes(arrow) were detected. Nuclear and cytoplasmic features fell short to define them as blasts.

2019,1 of the 2 devices used in our central laboratory created 7 blast/atypical lymphoid cells flags in 50 consecutive results. On that day, we validated a total of 915 complete blood count (CBC) test results. The next day, a total of 815 results were validated, and the same instrument again generated 7 blast/atypical lymphoid cells flags in 50 consecutive results. Of course it is impossible to determine an exact number of patients who

Address for correspondence: Ozgur Aydin, MD. Department of Biochemistry Laboratory, Kepez Public Hospital, Antalya, Turkey

Phone: +90 5432357629 E-mail: belozgur@hotmail.com ORCID: 0000-0002-6123-6186

Submitted Date: October 21, 2019 Accepted Date: November 04, 2019 Available Online Date: January 30,2020

${ }^{\circ}$ Copyright 2020 by International Journal of Medical Biochemistry - Available online at www.internationalbiochemistry.com

OPEN ACCESS This work is licensed under a Creative Commons Attribution-NonCommercial 4.0 International License. 
will require a microscopic examination, but these numbers are clues for estimated workload.

Since instrument flags are not sent to the LIS, the laboratory specialist validating the $C B C$ results and the clinician requesting the test may both be unaware of these warnings. However, the presence of blasts in a peripheral blood smear is a critical value to be reported. The $C B C$ is often used as a screening test in a public hospital like ours. Only a high index of clinical and laboratory suspicion leads to detection of blasts in blood samples of patients without salient clinical findings or a prior diagnosis. Each laboratory establishes its own manual review criteria. A hospital focused on cancer patients may take instrument flags into account much more seriously than a hospital visited by the general population [1]. Confirming the presence or absence of blasts in every flagged specimen would be impractical in most hospitals. It is not only a matter of cost-effectiveness, but a problem of trained and experienced staff. However, the clinical value of a true positive flag may be quite significant for the patient.

\section{Reference}

1. Barnes PW, McFadden SL, Machin SJ, Simson E; international consensus group for hematology. The international consensus group for hematology review: suggested criteria for action following automated $C B C$ and WBC differential analysis. Lab Hematol 2005;11:83-90. 